\title{
Application of Amplifluor-like SNP markers in plant genotyping
}

Shavrukov Y. ${ }^{*}$, Jatayev S. ${ }^{2}$, Kurishbayev A. ${ }^{2}$, Zotova L. ${ }^{2}$, Khassanova G. ${ }^{2}$, Baidyussen A. ${ }^{2}$, Langridge P. ${ }^{3,4}$, Soole K. ${ }^{1}$

${ }^{1}$ College of Science of Engineering, Biological Sciences, Flinders University, SA, Australia

${ }^{2}$ S. Seifullin Kazakh AgroTechnical University, Nur-Sultan, Kazakhstan

${ }^{3}$ School of Agriculture, Food and Wine, University of Adelaide, SA, Australia

${ }^{4}$ Wheat Initiative, Julius-Kühn-Institute, Berlin, Germany

*e-mail:yuri.shavrukov@flinders.edu.au

Single nucleotide polymorphisms (SNP) represent a very useful tool, used successfully in a wide array of plant genotyping projects. There are various methods for SNP analyses, most of which have been commercialised. The Amplifluor (Amplification with Fluorescence) SNP method is based on competitive allele-specific PCR, similar to that applied in KASP markers. Two steps are required to carry out Amplifluor SNP analyses: PCR using Universal probes (UPs) and then with Gene-specific primers (GSPs), which are developed independently. Each of the two UPs contains a fluorophore and a quencher with a 'hair-pin' fragment in-between. During PCR with a DNA template containing one of two alleles at the SNP position, the amplification with GSPs will result in the release of fluorescence from one of the UPs. The two UPs are relatively expensive, but their 'universality' allows for their purchase as a 'one-off' order that provides a stock for all further SNP analyses. This makes the application of the UP mixture much cheaper, since GSPs for each SNP cost the same as ordinary oligos. The significantly lower cost of Amplifluor-like SNP markers is accompanied by a high degree of freedom, completely 'in the minds' and 'in the hands' of researchers. Various SNP genotyping studies using self-designed Amplifluor-like markers were presented for analyses of many genes in different plant species. Examples include: Transcription factors DREB5 and NFYC-A7, and transcriptional repressor $D r l$ in wheat; intracellular vesicle trafficking genes, $R a b C$ GTP in chickpea; phytochrome C and SAP genes encoding stress-associated proteins in barley; chitinase genes in sugar beet; as well as a genetic polymorphism in different species of crested wheatgrass. All these cases provide useful 'fuel' for further candidate gene studies and Marker-assisted selection. 